\title{
Health information system as an integral component of cardiovascular surveillance system in Nepal
}

\author{
Sahadeb Prasad Dhungana ${ }^{1}$, Robin Man Karmacharya ${ }^{2}$, Prajjwal Pyakurel ${ }^{3}$,Archana Shrestha ${ }^{2,4,5}$, \\ Abhinav Vaidya ${ }^{6}$
}

${ }^{1}$ Nobel Medical College Teaching Hospital, Biratnagar, Nepal

${ }^{2}$ Dhulikhel Hospital, Kathmandu University Hospital, Dhulikhel, Kavre

${ }^{3}$ School of Public Health and Community Medicine, B.P. Koirala Institute of Health Sciences, Dharan, Nepal

${ }^{4}$ Harvard TH Chan School of Public Health, Boston, USA

${ }^{5}$ Institute for Implementation Science and Health, Dhulikhel, Nepal

${ }^{6}$ Department of Community Medicine, Kathmandu Medical College, Sinamangal, Kathmandu, Nepal

Corresponding Author: Sahadeb Prasad Dhungana

Nobel Medical College Teaching Hospital

Email:drsadhu@gmail.com

Cite this article as: Dhungana SP, Karmacharya RM, Pyakurel P. Health information system as an integral component of cardiovascular surveillance system in Nepal. Nepalese Heart Journal 2019; Vol 16(1), 7-10

Received date: 4th February 2019

Accepted date: 23rd March 2019

\section{Abstract}

Introduction: Nepal lacks a comprehensive, integrated health information system (HIS) to address the growing burden of cardiovascular diseases (CVDs).

Method: We performed a literature search and reviewed papers, government reports, and websites related to HIS. We included existing situations of HIS, major gaps, strength weakness opportunity threat (SWOT) analysis and role of different stakeholders to address CVD burden in Nepal.

Results: Health data from different health facility level are filled in district health information software (DHIS2). DHIS-2 has been implemented in 10 districts in full-fledged manner and partial phase in 22 districts. Data are collected by means of paper-based registers, tally sheets, and monthly data collation forms. The collated data are sent monthly to the district level and entered into the computer using DHIS-2 software and submitted to the national health departments.

Major gaps in health management information system (HMIS) are lack of separate heading of CVDs and lack of implementation of the existing data collection system. The strengths of the HIS are robust and decentralized health care delivery system in a good number of medical institutions. Weakness is lack of public and private partnership, concrete policy on health information and dissemination. Opportunities are the existence of policies and regulations mandating health facilities to report indicators, the involvement of private institutions and the expansion of existing DHIS-2 system.

Conclusion: Nepal currently lacks reliable and accurate data on timely manner to address the growing burden of CVDs. There is a need to strengthen the existing DHIS with a commitment from expertise and leadership.

Key words: Analysis, Cardiovascular diseases, Health information system

DOI: https://doi.org/10.3126/njh.v16i1.23890

\section{Introduction}

Cardiovascular disease (CVD) is the world's biggest killer claiming 17.7 million lives per year in 2015. Among the total deaths, one third prematurely occurs under 70 years of age. About $80 \%$ of all CVD deaths are from coronary heart disease and strokes. Around $75 \%$ of these deaths occur in developing countries. ${ }^{1}$

In Nepal, $42 \%$ of deaths are caused by non-communicable diseases (NCDs) and nearly $35 \%$ of deaths are caused by four particular NCDs - CVDs, cancer, chronic obstructive pulmonary disease, and diabetes mellitus. ${ }^{2}$ CVDs accounts for $17 \%$ of disease adjusted life years (DALY) among all the non-communicable disease and injuries (NCDI) in Nepal. ${ }^{3}$

Health information system (HIS) is an integral component of understanding country's capacity to integrate data collection, processing, reporting and use of information necessary for improving health services through better management at all level of health services. ${ }^{4}$

@Nepalese Heart Journal. Nepalese Heart Journal retains copyright and work is simultaneously licensed under Creative Commons Attribution License CC - BY 4.0 that allows others to share the work with an acknowledgement of the work's authorship and initial publication in this journal. 
Nepal health sector needs accurate, comprehensive and disaggregated data to gauge its performance, identify disparities between social groups and geographical areas and plan a further intervention. Work is already underway to make the recording and reporting system more accurate, comprehensive and userfriendly. These include revising HIS indicators, enabling the disaggregation of data, revising the data collection and reporting formats and strengthening the reporting process. ${ }^{5}$

Although the HIS for the communicable disease has been established in Nepal, the HIS for NCDs needs to be strengthened further. The information collected only from the government health system is not sufficient to establish the true burden of CVD in Nepal. The data collection, recording and reporting system of the private health sector at various levels needs to be strengthened. We aim to review the government's existing situation of HIS, identify major gaps of CVDs related HIS and explore the strengths, weakness, opportunities, and threats to establish CVDs surveillance system in Nepal.

\section{Method}

We performed a literature review and identified different areas under which the search strategy was based. This included existing situations of HIS, major gaps in HIS for management and prevention of CVDs, strength weakness opportunity threat (SWOT) analysis, HIS and role of different stakeholders to address CVD burden in Nepal. We reviewed different papers, government reports, other relevant reports and websites related to HIS to collect the information. We conducted a Skype call discussion with our all investigators once a week and summarized our findings. In addition, we visited the Nepal Health Research Council (NHRC) office, an apex body of research in Nepal at Ram Shah Path, Kathamandu, Nepal and conducted key informant interview with the research officer. Similarly, we visited management information section (MIS) at Department of Health Services (DOHS) at Teku, Kathmandu, Nepal and conducted in-depth interviews with the statistician and few administrative personnel working in that department. HMIS at DOHS is the key department which looks after all the HIS throughout the country.

\section{Results}

\section{Existing situation of HMIS in Nepal}

Cardiovascular HIS include data and information on individuals with CVDs and the overall Nepalese population. DOHS have implemented the HIS in the entire country since 1994. The overall purpose of the DOHS is to deliver preventive, promotive and curative health services throughout Nepal. The DOHS is one of three departments under the Ministry of Health. Health data from different health facility level are filled in District Health Information Software (DHIS). It is an open source software platform for reporting, analysis, and dissemination of data for all health programs. The DHIS consists broadly of two parts: data collection and analysis which occurs in the facility level and DHIS software which processes data. DHIS is used in more than 60 countries around the world. The electronic version being used in Nepal is DHIS-2. All the statistician and medical recorder in every district have been trained to use this software. DHIS-2 has been implemented in 10 districts in full-fledged manner and partial phase in 22 districts. Data are collected on all services provided by a facility level. These are collected by means of a paper-based system of registers, tally sheets, and monthly data collation forms. The collated data are sent monthly to the district level where they are entered into the computer using DHIS 2 software then analyzed and a report is submitted to the district and national health departments.

\section{Major gaps in HIS for the prevention and manage} ment of CVDs in Nepal

There is no separate heading of CVDs in HIS system in Nepal, although Management Information Section (MIS), DOHS uses ICD-10 data coding system to collect the data. However, there is a huge limitation on its adherence even by many governments, semi-government hospitals and private medical colleges causing an incomplete representation of data. In order to address CVDs, there should be a separate component of CVDs or NCDs in HIS of the government of Nepal. Similarly, annual health report published by DOHS should address separate chapter for CVDs or NCDs similar to a chapter entitled "disease control" where the details of many infectious disease and zoonotic disease are addressed. ${ }^{5}$

\section{SWOT analysis}

The strengths are robust and decentralized health care delivery system from center to the grassroots level and a good number of government and private hospitals, medical colleges, teaching center, international and national non-governmental organizations. Weakness is lack of public and private institutional partnership, lack of concrete policy on CVDs health information and dissemination, lack of an effective program on organizational capacity building to develop competency and resources in health information and concrete plan for monitoring and evaluating the burden of CVDs. Opportunities are the existence of policies, laws, and regulations mandating public and private health facilities to report indicators, sustaining interest among different stakeholders for the continuous development of HIS, involvement of rampantly growing private hospitals and medical colleges, expansion of existing DHIS-2 system at all health care delivery system. Threats are a dearth of health professional trained in CVDs, poor coordination of public and private health sectors, uneven distribution of health care system, poor implementation of laws and policies, lack of adequate funding for capacity building and human resources, lack of political commitment and corruptions in the health sector.

\section{Role of different stakeholders to address CVDs in Nepal}

Every sector has a role to play to address CVD burden in Nepal. Ministry of Health and Population is the highest governing body for health care should take a stake in the implementation of plans and policies related to CVDs. Similarly, MIS should focus on developing strong reporting of CVDs data and its timely dissemination. Public and private health care sectors should provide a robust mechanism of collecting, reporting and recording data. Health care providers should be skilled in the clinical diagnosis of CVDs and finally, the public needs to be cautious about their cardiovascular health and timely checkup for prevention of CVDs.

\section{Discussion}

Information plays a key role in management, planning, monitoring, evaluation and policy development related to CVDs. Nepal currently lacks a comprehensive, integrated system to address the growing burden of CVD, including the reliable and reliable and comprehensive data on timely manner that can be used by policymakers, health care providers, researchers, and the public. ${ }^{6}$ Although, sophisticated information regarding 
cardiovascular health and diseases are available, from prevention to advanced care and treatment. However, it is the coordination of data collection, standards, and information that is the key to success in planning and providing care.

CVDs are the major public health challenges in the 21st century, being the most common among NCDs in Nepal. Though there is no routine surveillance or registry system and actual burden and trend of CVDs remain uncertain in Nepal, NCDs survey done in 2007 and STEPS survey in 2013 has shown conventional risk factors to be high among Nepalese population. ${ }^{7,8} \mathrm{~A}$ hospital-based study of NCDs across the country has shown that $36.5 \%$ of the admissions were for NCDs, of which $38 \%$ cases were CVDs. ${ }^{9}$ Though these are a population-based survey conducted through sampling, strong HIS for CVDs is the need of the hour to estimate the true burden of CVDs and apply the intervention packages. Additionally, strong HIS data could be used to inform the public and decision-makers for planning and evaluating prevention and control programs and designing health policy and legislation for CVDs in Nepal.

Worldwide, there are surveillance systems which have addressed the risk factors of CVDs. ${ }^{10}$ The Behavioral Risk Factor Surveillance Systems (BRFSS) was established by the Centers for Disease Control and prevention to assess health-related risk behavior, chronic health condition and use of preventive services (CDC,Atlanta, USA) making it the largest telephone health survey in the world. ${ }^{11}$ World Health Organization (WHO) formulated the Global STEPwise approach for NCD risk factor surveillance aimed at collecting data on risk factors in a stepwise manner according to the complexities involved that would be comparable across variable sites in the world. ${ }^{12}$ Similarly, the Indian Council of Medical Research (ICMR) - WHO collaborative initiative on NCD risk factors surveillance provided the requisite experience and mandate to respond to the call of the Ministry of Health and Family Welfare, Government of India, to develop the strategy and modules for undertaking NCD risk factor surveillance at a national level. ${ }^{13}$

Surveillance system similar to other countries can be established in Nepal and the collected data can be linked to policy development and interventions. Different stakeholders including government representatives, local authorities, public health workers, academicians, and researchers need to foster partnership and collaboration for an effective CVD surveillance system in Nepal.

\section{Major strengths}

This review highlights the present scenario of the growing burden of CVDs in Nepal and the status of HIS on CVDs. Identification of major gaps and SWOT analysis helps to focus on HIS on CVDs and work on it for improvement in the future. These may be useful to sensitize health planners and policymakers about the importance of HIS on CVDs. Annual health report published by DOHS should address separate chapter for CVDs similar to a chapter entitled "disease control" where the details of many infectious disease and zoonotic disease are addressed. ${ }^{14}$

\section{Weakness}

This review was prepared from limited information and resources obtained from different papers, government reports, websites related to HIS and interview with key informants. This may not provide complete information about HIS on CVDs. Moreover, the scenario of HIS on CVDs at private health institutions and problems faced by them has not been included.
The real scene of implementing HIS on CVDs at the grass-roots level and their capability in diagnosing and reporting such data has not been ascertained in this review.

\section{Recommendation}

There is a need to accelerate the process of developing electronic medical records (EMRs) with the strengthening of existing DHIS-2 across all districts, provinces with public and private partnership. Enhanced efforts are needed to provide timely and appropriate information which is culturally and ethnically appropriate for cardiovascular health promotion, care, and services. Existing databases, surveys, and registries should be pooled and linked to produce a periodic national cardiovascular health report which will help to improve the patient care and policy making. Challenges pertaining to human resources and funding must be addressed by the development of an education and capacity building training for health care providers and allocation of part of the health budget to strengthen information on CVDs.

\section{Conclusion}

Good and inclusive HIS will act as a foundation for good health system and performance. Nepal currently lacks reliable, comprehensive and user friendly data to address the growing burden of CVDs. This needs a robust HIS. Better representation of CVDs in HIS is of utmost importance to address the rising burden of CVDs in Nepal. A sustained integrated system requires a major commitment of expertise, leadership, and funding. There is a need to strengthen the existing DHIS and monitor targeted program across the country.

\section{Conflict of interest}

The authors declare no conflict of interest in the publication of this article.

\section{Acknowledgements}

We are grateful to the National Heart, Lung and Blood Institute (NHLBI), National Institute of Health (NIH),USA (project number: 1U24HL136789-01) for providing support in capacity building initiative to address CVDs in Nepal. We would like to acknowledge the support of Nepal Health research council and Management Information Section, Department of Health services, Nepal for providing valuable information about HIS on CVDs. We would also like to acknowledge the Principal investigator of this project Dr. Rajendra Koju, Co-investigators Dr.Rajeev Shrestha, Dr. Biraj M Karmacharya, Dr. Natalia Oli for their support.

\section{Reference}

1. World Health Organization. Cardiovascular Disease [Internet].Cited [2018 August 9]. Available from:

http://www.who.int/cardiovascular_diseases/en

2. Nepal Health Research Council (NHRC): NonCommunicable Diseases Risk Factors: STEPS Survey Nepal 2013. Kathmandu. NHRC 128p.

http://www.searo.who.int/nepal/mediacentre/non_ communicable_diseases_risk_factors_steps_survey_ nepal_2013..pdf

3. The Lancet NCDI Poverty Commission Nepal. An Equitable Initiative to Address Non-Communicable Disease and Injuries. Kathmandu 2018 March 26.56p 
https://static1.squarespace.com/static/55d4de6de4b011a1 673a40a6/t/5abe6570f950b72797ffe25a/1522427249521/

Nepal+Commission+Report+Cover. pdf

4. Health Systems 20/20. 2012. The Health System Assessment Approach: A How-To Manual. Version 2.0. www.healthsystemassessment.org

5. Strengthening the health Management Information system: Responding to the need for improved and disaggregated data [online]. Cited [2018 October 20].

http://nhsp.org.np/wp-content/uploads/2016/08/FINALPulse-HMIS-12th-Nov-2013.pdf

6. Vaidya A. Tackling cardiovascular health and disease in Nepal: epidemiology, strategies, and implementation. Heart Asia 2011; 3(1): 87-91.

7. Ministry of Health and Population, Nepal. Noncommunicable disease risk factors survey 2007, Kathmandu, Nepal: Ministry of health and population, 2009

8. Non-communicable diseases risk factors: STEPS survey Nepal 2013.

http://www.searo.who.int/nepal/mediacentre/non_ communicable_diseases_risk_factors_steps_survey_ nepal_2013..pdf

9. Nepal Health research council. Prevalence of Non -communicable diseases in Nepal: Hospital-based study. Kathmandu, Nepal. Nepal Health research council, 2010.

10. Shah B and Mathur P. Surveillance of cardiovascular disease risk factors in India: The need \& scope. Indian J Med Res. 2010; 132(5): 634-642.

11. CDC 2004a. Behavior Risk Factor Surveillance System. Atlanta, GA: Centers for Disease Control and Prevention. Available: http://www.cdc.gov/brfss

12. World Health Organization. Noncommunicable Diseases and Mental Health Cluster. (?2005)?. WHO STEPS surveillance manual : the WHO STEPwise approach to chronic disease risk factor surveillance / Noncommunicable Diseases and Mental Health, World Health Organization. World Health Organization. http://www.who.int/iris/handle/10665/43376

13. Report of the ICMR - WHO study on assessment of the burden of non-communicable diseases. New Delhi: Indian Council of Medical Research; 2006.

14. Annual Report, Department of Health Service 2073/2074 (2017).

https://dohs.gov.np/wp-content/uploads/2018/04/Annual_ Report_2073-74.pdf 